\title{
GD3 Binding
}

National Cancer Institute

\section{Source}

National Cancer Institute. GD3 Binding. NCI Thesaurus. Code C40955.

GD3 Binding Interaction involves non-covalent attachment of a molecule through intermolecular physical forces of attraction to a cell surface liposaccharide disialog ang lioside, GD3. 